\section{Hyperfine Structure and Saturation Effects in the Paramagnetic Resonance Spectrum of Manganese}

A Microwave bridge in conjunction with a superheterodyne method of reception has been used for the detection and measurement of weak para. magnetic resonances. It has been possible to investigate, at a frequency of $9,501 \cdot 5 \mathrm{Mc}$./sec., the resonance spectrum of $\mathrm{Mn}^{++}$ions contained, in concentrations down to 1 part in $10^{5}$, as activators in zine sulphide phosphors.

By means of a $50 \mathrm{c} / \mathrm{sec}$. field modulation the magnetic absorption at room temperature is displayed as a function of magnetic field. For the specimen with a concentration of 1 per cent manganese the resonance absorption curve is similar in shape but narrower (width approximately 300 gauss) than that for hydrated manganese sulphate crystals ${ }^{1}$. A reduction of 'exchange narrowing' may account for the greater overall width of the resonance observed for the next lower concentration of 0.1 per cent manganese. The more interesting feature of the decrease in concentration is, however, the appearance of a hyperfine structure shown by a splitting into six peaks in agreement with the spin of $5 / 2$ for the manganese nucleus. An additional structure is just detectable at this concentration.

The spectrum for the lowest concentration $(0.001$ per cent Mn) is shown in Fig. 1 and has been examined in more detail by reducing the sweep amplitude and photographing in sections for different static mag. netic fields. The complete structure is reproduced diagrammatically in Fig. 2; the magnetic fields corresponding to the individual peaks have been obtained by calibration against proton resonance.

The six main peaks of the complex spectrum are spaced at intervals of $66 \cdot 2,66 \cdot 9,67 \cdot 6,68 \cdot 3$ and $71 \cdot 5$ gauss, corresponding to a hyperfine structure interval of $0.006 \mathrm{~cm} .^{-1}$, of the same order as that obtained for copper and cobalt ${ }^{2,3}$. The interpolated centre of this series lies at $3,384 \cdot 2 \pm 0 \cdot 5$ gauss and corresponds to a $g$ factor, after correction for the shift introduced by the hyperfine splitting, of $2(1 \cdot 0012 \pm 0 \cdot 0002)$. This figure agrees satisfactorily with the electron spin $g$ value of $2(1.00119 \pm 0.00005)$ obtained by Kusch and Foley ${ }^{4}$ from atomic beam experiments. The subsidiary structure of the spectrum results from a second-order splitting of the ${ }^{6} S_{5 / 2}$ ground state of $\mathrm{Mn}++$ by the crystalline electric field ${ }^{5}$. In the absence of detailed information on field symmetry and measurements with single erystals, no detailed analysis is possible; but the diagram indicates a possible grouping into five series, corresponding to an initial splitting of the order of $0.002 \mathrm{~cm} .^{-1}$.

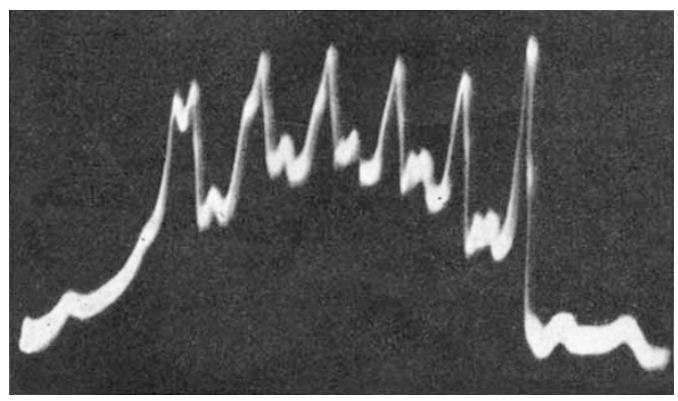

Fig. 1
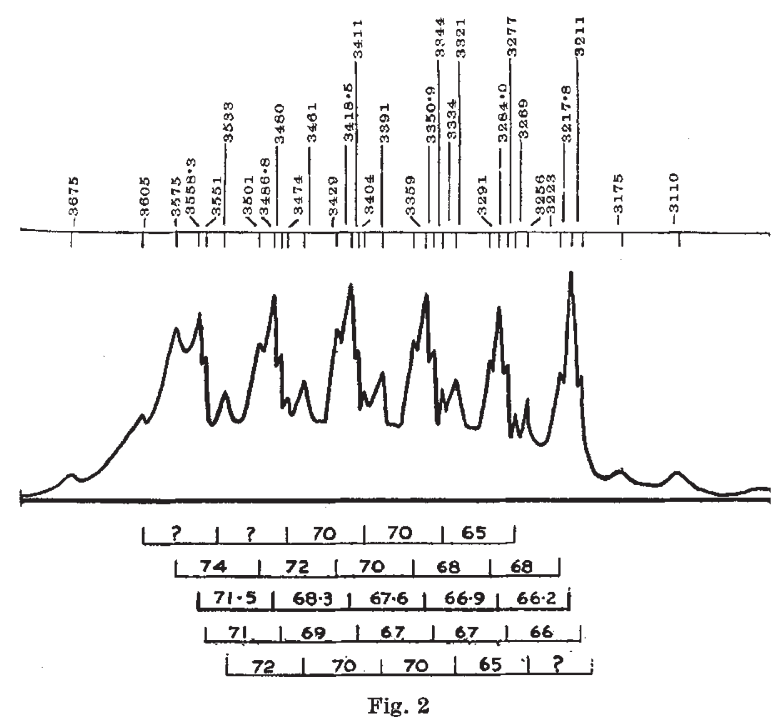

Experiments at liquid-air temperature, using the phosphor with the lowest manganese concentration, have shown that the general structure of the spectrum is not materially different from that at room temperature, which indicates that the width of the lines is predominantly determined by spin-spin interaction. However, the low-temperature measurements have yielded information on the spin-lattice relaxation. In contrast with the high powers required for investigations on paramagnetic salts ${ }^{6}$ the power-level available from the reflex klystron ( $100 \mathrm{~mW}$.) has been adequate for the study of relaxation effects in the magnetically dilute phosphor. A saturation effect, indicated by a decrease with increasing input of the ratio of magnetically absorbed power to input power, has been clearly established, and from quantitative measurements a value for the spin-lattice relaxation time of $10^{-8}$ sec. has been obtained.

We are greatly indebted to Dr. G. F. J. Garlick, of the University of Birmingham, for the supply of phosphor specimens.

\section{T. S. ENGLAND}

E. E. SCHNEIDER

King's College,

University of Durham,

Newcastle upon Tyne.

${ }^{1}$ Cummerow, R. L., Halliday, D., and Moore, G. E., Phys. Rev., 72, 1233 (1947).

${ }^{2}$ Penrose, R. P, Gorter, C. J., Abragam, A., and Pryce, M. H. L., Nature, 163, 992 (1949). Bleaney, B., Ingram, D. J. E., and Pryce, M. H. L., Nature, 164,
116 (1949).

- Kusch, P., and Foley, H. M., Phys. Rev., 74, 250 (1948).

'Van Vleck, J. H., and Penney, W. G., Phil. Mag., 17, 961 (1934).

- Slichter, C. P., and Purcell, E. M., Phys, Rev., 76, 466 (1949).

\section{Gelation of Montmorillonite}

THE combination of optical and elastic studies on sols of Wyoming bentonite containing the smallest particle sizes (15-20 $\mathrm{m} \mu$ equivalent spherical diameter) have led to the conclusion that the gelation of this system is due to the formation of a fibrillar network in which the plates are aggregated end-to-end in the form of flat, ribbon-like filaments. The growth of these aggregates is indicated in Fig. 1, which shows the increase in the dissymmetry coefficient of the scattered light, and hence an increase in the size 